\title{
EFFICIENT DATA GATHERING BY MOBISINK IN WSN USING OPTIMIZED ROUTE POINTS INTELLIGENCE SYSTEM (ORPIS)
}

\author{
KUMAR SWAMY. B. $\mathbf{V}^{\mathbf{1}}$, GOWRAMMA. Y. P $^{2}$ \& ANANDA BABU. J ${ }^{3}$ \\ ${ }^{1}$ Robert Bosch Engineering and Business Solutions, Bangalore, Karnataka, India \\ ${ }^{2,3}$ Kalpataru Institute of Technology, Karnataka, India
}

\begin{abstract}
Evolution of mobile sink(MS) has gained significant importance in the wireless sensor network(WSN). With the increase in demand of sensor network technology, it is important to maintain the QoS parameters to meet the requirements of the intended application. Several studies have been done to improve the energy usage of the sensor network and latency. Harvesting of energy to sensor nodes is helpful in field areas related applications. However, finding best route path of MobiSink node is a challenging. In this paper, a new protocol is derived which introduces gaining of self-knowledge by MobiSink based on network behavior. Optimized route points are calculated based on prediction analysis by MobiSink. Data generation rate of source nodes helps in finding the route paths frame duration for MobiSink which improvises the data gathering. A protocol is simulated with NS2 tool and the results show better performance of the proposed protocol.
\end{abstract}

KEYWORDS: Energy Consumption Rate (ECR),Energy Harvesting Rate (EHR) \& Route Points(RP)

Received: May 11, 2018; Accepted: Jun 01, 2018; Published: Jun 30, 2018; Paper Id.: IJCNWMCAUG20181

\section{INTRODUCTION}

Wireless Sensor Networks (WSNs) consist of low-cost sensor nodes which collect data from the environment and relay them to a sink where they are subsequently processed. WSNs are employed in wide range of applications such as security surveillance, battlefield, intrusion detection, target tracking purposes etc. Deployment of a large set of sensor nodes specific to application makes them impossible to hand place where battery replacement is usually cumbersome (especially in harsh environments like battlefields) [1]. Being functional for a prolonged period is its fundamental objective. Usually in many-to-one multi-hop WSNs, the sink's one-hop vicinity nodes most often 'funnel' (forward) data on behalf of all other nodes. Clearly, network in which data collection rate dominates data forwarding rate (in a traffic intense application, e.g. Video-based target tracking), typically congestion builds up at the bottleneck nodes (nodes at the sink's one-hop neighborhood). Consequently, packet dropping incident and/or retransmission becomes more frequent, leading to increasingly degraded network performance. Additionally, hotspot (sink's one-hop neighbor) nodes die earlier (they exhaust their energy as they forward higher volume of traffic compared to other nodes) relative to other nodes in the network. Energy depletion to hot spot nodes causes network partitioning, leading to complete isolation of the sink node resulting in an entire network failure. Hence this hot spot problem must be adequately dealt with, through measures that prevent the failure of the sink's one-hop neighbors by reducing load caused to these nodes. 
Applying an efficient mobile sink strategy into the multi-hop routing protocols is an effective solution that tends to prevent network partitioning in WSNs [2]. Instead of replacing these hotspot nodes, the key idea is to move the sink periodically to various parts of the network with sufficient energy for data gathering. During the sink' trajectory, as the sink's one-hop neighbors keeps changing in time, the energy consumption and the traffic load (for sink's neighbor nodes) could be balanced all over the network.

This mechanism consequently increases the network lifetime [3]. Thus, optimization for energy consumption is foreseen as an important problem, especially to prolong network lifetime in WSNs [4]. The use of sink mobility in WSN is commonly recognized as one of the most effective means of load balancing, ultimately leading to fewer failed sensor nodes and longer network lifetime. This also enhances reliability, accuracy, flexibility, cost-effectiveness and ease of deployment [5]. Recent research on data collection reveals that gathering sensor data from error-prone routes (long and multi-hop routes) to a static sink, leveraging sink mobility for data gathering is more promising for energy efficient data gathering [6]. The main challenge of this technique is the difficulty is dynamic route discovery of the mobile sink to travel across the network, such that the data collected from sensor nodes are within specific time.

Applying an efficient mobile sink strategy [2] into the multi-hop routing protocols is an effective solution that tends to prevent network partitioning in WSNs. Instead of replacing these hotspot nodes, the key idea is to move the sink periodically to various parts of the network with sufficient energy for data gathering. During the sink' trajectory, as the sink's one-hop neighbors keeps changing in time, the energy consumption and the traffic load (for sink's neighbor nodes) could be balanced all over the network.

This mechanism consequently increases the network lifetime [3]. Thus, optimization for energy consumption is foreseen as an important issue, especially to prolong network lifetime in WSNs [4]. The use of sink mobility in WSN is commonly recognized as one of the most effective means of load balancing, ultimately leading to fewer failed sensor nodes and longer network lifetime. This also enhances reliability, accuracy, flexibility, cost-effectiveness and ease of deployment [5]. Recent research on data collection reveals that gathering sensor data from error-prone routes (long and multi-hop routes) to a static sink, leveraging sink mobility for data gathering is more promising for energy efficient data gathering [6]. The main challenge of this technique is the difficulty is dynamic route discovery of the mobile sink to travel across the network, such that the data collected from sensor nodes are within the specific time period.

This paper presents Efficient Data Gathering by MobiSink in WSN using Optimized Route Points Intelligence System (ORPIS) protocol which will be concentrate on two different issues like:

- Energy Harvesting Model: Renewal of energy from surroundings such solar cells is used to derive an energy harvesting model. Certain assumptions are made and stated below.

- Tour Length Model: Traversing of MobiSink node is to reach every source node within the network and collect data from each.

The detail explanation is provided in the proposed methodology, The paper contains related work, proposed methodology and algorithm used for the research and simulation parameters taken for the analysis. 


\section{RELATED WORK}

One of the major disadvantages in using mobile sink is the increased latency caused during data collection due to the speed of the mobile sink typically ranging between $0.1-2 \mathrm{~m} / \mathrm{s}[7]$ [8].

In a single-hop approach, the distance between the sink and the source is only one hop. The mobile sink visits each sensor node and gathers its data, apparently, in such networks the energy consumption of sensor nodes are minimized (as communication is done using only one hop),

However, the expense of high data delivery delay and possibility that mobile sink may visit only some locations of the WSN is its drawback. Consequently, these types of approaches are more suitable for delay-tolerant networks. Many other research works have been proposed on multi-hop data gathering approach using the mobile sink.

In Cluster based strategy [9], the network is divided into clusters. Each cluster is associated with a cluster head, which is responsible for gathering data from sensor nodes, aggregating and transmitting them to the mobile sink. In this type of approach, the sink consistently updates its current location to its nearest cluster head, which is in turn communicated through control message to all other cluster heads in the network. Additionally, cluster head should constantly be kept updated about the mobile sink's current location thereby creating considerable routing overhead.

In Adaptive Reversal Tree (ART)[10], the tree is constructed to learn the route of mobile sink. A major drawback of this approach lies in updating the whole network with the current position of the sink. The tree reconstruction cost becomes higher when the affected area increases. While in distributed tree-based data dissemination (TEDD) [11]approach, the new position of the sink was known only to the one-hop neighbors, which leads to the less control packet overhead for effectively and efficiently managing the sink mobility, but finding on optimal data gathering tour in general becomes a hard problem. Due to constrained access areas or obstacles in the deployed field, the problem of finding the sink movement path to optimize the lifetime of the WSN is hard to solve and pose more complexity [12]. Additionally, the problem concerning mobile sink to avoid data loss due to sensor buffer overflow causing high latency in existing approach was further evaluated and thus the proposed work was designed to enhance effectiveness in mobile sink data gathering methodology with reduced latency and prolonged network lifetime.

The author in [11] describes the various mobile elements has created a new dimension to reduce and balance the energy consumption in wireless sensor networks. However, data collection latency may become higher due to the relatively slow travel speed of mobile elements. Thus, the scheduling of mobile elements, i.e., how they traverse through the sensing field and when they collect data from which sensor, is of ultimate importance and has attracted increasing attention from the research community. Formulated as the traveling salesman problem with neighborhoods (TSPN) and due to its NP-hardness, so far only approximation and heuristic algorithms have appeared in the literature, but the former only have theoretical value now due to their large approximation factors. In this paper, following a progressive optimization approach, we first propose a combine-skip-substitute (CSS) scheme, which is shown to be able to obtain solutions within a small range of the lower bound of the optimal solution. We then take the realistic multi-rate features of wireless communications into account, which have been ignored by most existing work, to further reduce the data collection latency with the multi-rate CSS (MR-CSS) scheme. Besides the correctness proof and performance analysis of the proposed schemes, we also show their efficiency and potentials for further extensions through extensive simulation. 


\section{PROPOSED WORK}

Efficient collection of data by MobiSink node requires a well-defined path trajectory allowing proper setup of time frames at each location. Design of protocol is to analyze the appropriate data points within the network where the data collection is maximum. It means, MobiSink node should have the proper knowledge to make decisions dynamically. Several factors are considered to design such a system. Amount of time needed for every location where data is available is studied and is the basis to design the protocol. Selection of such location points should be within the transmission range of MobiSink and source node.

With all the above points, certain assumptions are derived. A network is designed to be consisting of $N$ nodes deployed within the network area of $(X, Y)$. Deployment of source nodes set to random which brings real-time scenario. There might be a possibility that few source nodes may fall within transmission range of each other and those together will form a group $S G_{i}$. An occurrence of $S G_{i}$ helps in deriving a mean point within their surroundings. And, that point will be shared with mobisink which becomes route point $R P_{i}$ to mobisink.

Sensors are also equipped with renewable energy device and hence, the algorithm is derived to incorporate such system. Node is said to have harvested energy $H_{i}$ at any point in time. The energy of the system will run for long time and lifetime of the network will be extended. Also, the reliability of the system will have a direct relationship with the energy of the network. So, higher reliability can be achieved without negotiating with the energy of the node.

History tracking of tour length information is required which helps to decide provisioning of the time frames $t f_{i}$ at each $R P_{i}$. The Algorithm is derived to introduce intelligence to the system with to formulate an optimistic $T L$ for every next iteration with the previous history.

\subsection{Problem Definition}

Gathering of data in wireless sensor network generated from many sensor nodes must fit for certain QoS. Energy efficiency and latency are the key QoS factors that decide whether the network is suitable for real time. With mobisink which traverses across the network will have to meet the above QoS. Traveling of mobisink leads to loss energy to travel and delay in collecting data. To overcome these problems, a protocol is designed. Two models are designed which gives solution for both energy and latency. And, those are studied below.

- $\quad$ Energy Harvesting Model

- $\quad$ Tour Length Model

\subsubsection{Energy Harvesting Model}

Renewal of energy from surroundings such solar cells is used to derive an energy harvesting model. Certain assumptions are made and stated below. A node assumed to be having storing energy capacity of $C$ and energy stored at given time interval is $P$. With the energy renewable model $H$, energy at any time $P_{i}(e)$ is defined using (1),

$P_{i}(e)=\min \left\{P_{i-1}(e)+H_{i-1}(e)-D_{i-1}(e), C(e)\right\}$

where, $H_{i-1}(e)=$ harvested energy,

$D_{i-1}(e)=$ depleted energy, 
$P_{i}(e)=$ stored energy at each node

With the assumption as given (2)

$0 \leq P_{i}(e) \leq C(e)$

\subsubsection{Tour Length Model}

Traversing of mobisink node is to reach every source node within the network and collect data from each. Mobisink should reach till the point where it falls within transmission range of source node. With multiple source nodes within the specified area should accept the criteria mentioned in (3) to have a route point $R P$.

$$
R P \exists \operatorname{dist}\left(n_{s_{i}}, n_{s_{j}}\right)<r_{c}
$$

where, $r_{c}=$ radio range

$n_{s_{i}}=$ source node $i$

If any such $R P$ are present, then such points are derived using (4)

$$
R P_{i}=\frac{\sum_{i=0}^{k} n_{s_{i}}}{k}, \text { wherek }=\text { countofelementsinS } G_{i}
$$

where, $S G_{i}=$ source node group

Tour length $T L$ is the total amount of time required for mobisink to traverse in the network to collect data from all source nodes. Hence, it is a combination of traveling time and time spent at each $R P_{i}$ to collect data and given below in (5). Time spent at each $R P_{i}$ is termed as time frame $t f_{i}$.

$$
T L=\sum_{i=0}^{n} t f_{i}+\sum_{i=0}^{n} t_{R P_{i}}, \text { where } n=\text { count of all } R P S
$$

\subsubsection{Intelligent System}

With above two models, the mobisink node is equipped with a knowledge system which will have the capability of finding data collection time interval. The node having higher harvesting energy rate of the previous iteration will have a higher amount of data to be transmitted to mobisink. With such system is taken into assumption, the model is derived using (6)

$$
t f=\frac{e_{b}}{e h_{i-1}}
$$

where, $e_{b}=$ energy spent per each byte

$e h_{i}=$ harvested energy rate

If there is a $S G_{i}$ then, equation (6) is revised by considering average harvesting rate $e h_{\text {avg }}$ and multiplying with the total number of nodes within $S G_{i}$ using (7)

$$
t f_{i}=\frac{n_{S G_{i}} e_{b}}{e h_{\text {avg-1 }}}
$$

\subsection{Proposed Algorithm}

Algorithm for the above defined mathematical model is defined here. The network will consist of normal nodes, 
source nodes and mobisink node. Figure 3.1 covers the complete algorithm flow for the proposed algorithm. Nodes are deployed using randomly using uniform distribution. After deployment, source nodes are selected at random. Mobisink has to collect the location information of all source nodes within the network which is required to find route points in the network. Therefore, source node broadcast their location information to mobisink. Intermediate nodes ensure that update information to mobisink.

All location points are mapped to find route points to reduce the number of visiting points by mobisink in the network. If more than one source nodes are within transmission range of each other, then their mean position will be selected as route point. Route points calculated thus will be maintained in a vector to find time frame for data collection.

Intelligence system will iterate through all the route points obtained. With the help of harvested energy rate obtained in previous iteration and energy spent for transmission of each byte, the time frame of the next iteration will be calculated. One previous history information is sufficient to find the time frame. Thus, store size required will be minimal. Also, since the computation is simpler, the computation energy will not add overhead to the system.

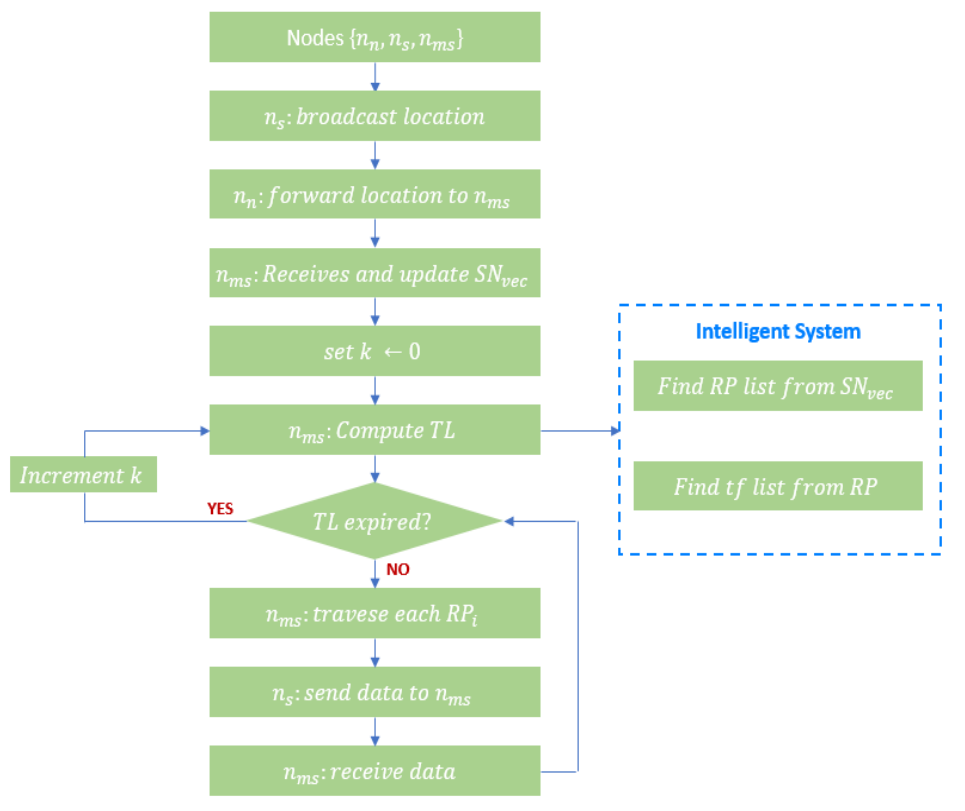

Figure 3.1: Algorithm of ORPIS Protocol

A detailed flow of intelligent system designed for mobisink node is shown in figure 3.2. Nodes falling within same transmission range will be put it in a temp list. Finally, mean value will be calculated which becomes a route point for those source node groups. With these route points, the time frame is calculated with average harvesting energy rate and energy spent for transmission of each byte. 

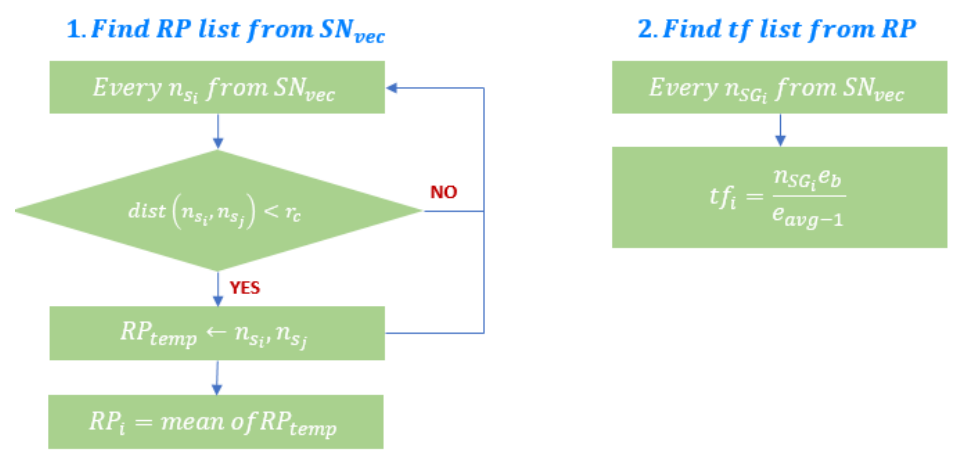

Figure 3.2: Intelligent System - Route Point and Time Frame Algorithm

\section{PERFORMANCE ANALYSIS}

\subsection{Simulation Parameter}

Table1: Simulation Parameters

\begin{tabular}{|c|l|l|}
\hline Sl. No. & \multicolumn{1}{|c|}{ Parameter } & \multicolumn{1}{c|}{ Values } \\
\hline 1 & Network size & $(50-150)$ \\
\hline 2 & Packet transmission rate & $250 \mathrm{Kbps}$ \\
\hline 3 & Packets generation rate & 10 packets per second \\
\hline 4 & Maximum transmission range & $200 \mathrm{~m}$ \\
\hline 5 & Amount of energy collected by $\mathrm{EH}$ & $\begin{array}{l}655.15 \mathrm{mWh} \text { (sunny day) } \\
313.70 \mathrm{mWh} \text { (cloudy day) }\end{array}$ \\
\hline 6 & Solar panel size & $10 \mathrm{~mm} * 10 \mathrm{~mm}$ \\
\hline 7 & Ambient temperature & $31.8^{\circ} \mathrm{C}$ \\
\hline
\end{tabular}

\subsection{Remaining Energy}

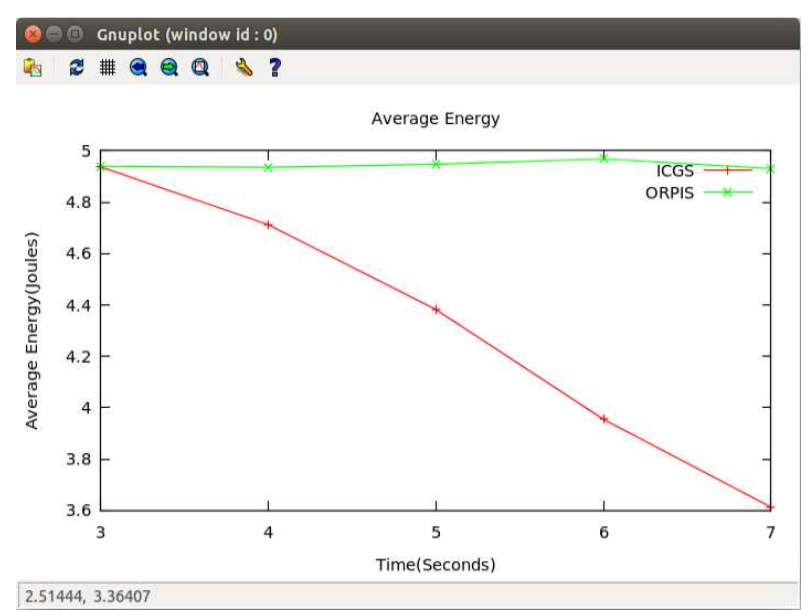

Figure 4.1: Remaining Energy of Source Nodes

Figure 4.1 shows the remaining energy of source nodes within the network. The results are compared with ICGS protocol. Participation of nodes in data transmission is proportionated with their energy replenishment as compared to ICGS where renewal energy and node participation in data transmission has not related each other. Result show that nodes will maintain their energy almost same throughout the simulation and is nearly equal to their initial energy. From the results, it is found that energy of ICGS will reduce to $12 \%$ of ORPIS protocol after about 7 seconds after simulation begins. 


\subsection{Data Collection with Variable EH Rate}

Purpose of the protocol is to increase the efficiency of data collection. Therefore, the collection of data is plotted in figure 4.2. This result considers a number of source nodes set to 8 . And, harvesting of energy is varied between (313.70 $\mathrm{mWh}, 655.15 \mathrm{mWh}$ ). Though IMPR also posses harvesting of energy, ORPIS ensures that proper amount of time spent at each visiting place to collect all data from the respective positioned source nodes. Thus, there is an average of $22 \%$ increase in the data collection in ORPIS protocol compared to proposed IMPR protocol.

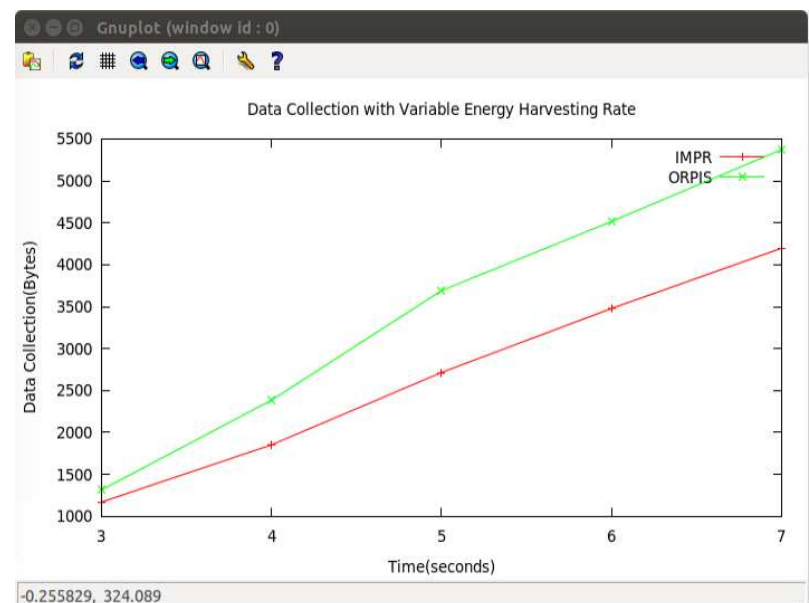

\section{Figure 4.2: Data Collection with Variable Energy Harvesting Rate}

\subsection{Data Collection with Constant EH Rate}

Data collection results further tested with constant harvesting energy rate $655.15 \mathrm{mWh}$ and the results are plotted in figure 4.3. With constant sunny day, data collected will be higher as high reliability is achieved with higher energy investment. This results in further improvement in the results and an improvement factor of $34 \%$ is achieved with constant energy harvesting rate.

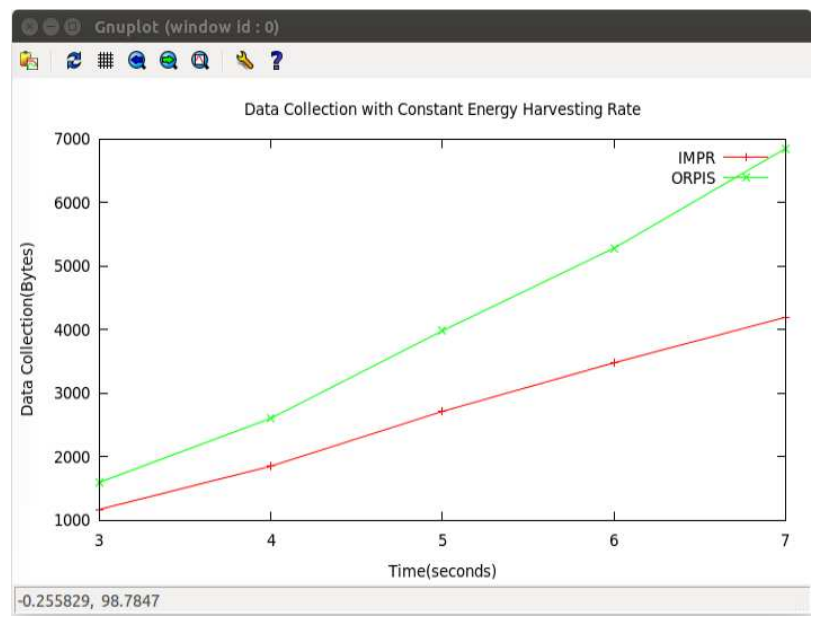

Figure 4.3: Data Collection with Variable Energy Harvesting Rate 


\subsection{Packet Delivery Ratio}

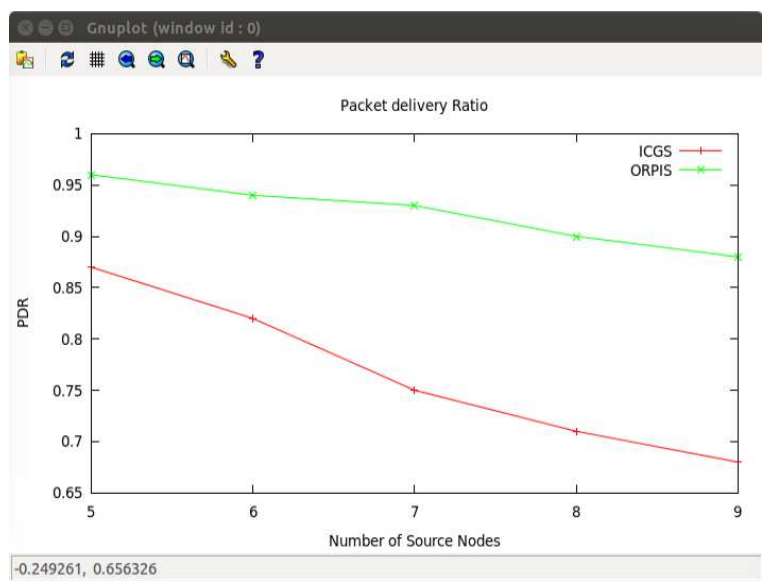

Figure 4.4: Packet Delivery Ratio

Figure 4.4 shows the results of packet delivery ration with respect to source nodes. Optimistic selection of visiting points and proper allocation of time frames at each point has improved the receiving ration of packets by mobisink. Results will even better performance if source nodes are nearby each other. Reduction of delivery ration with a higher number of source nodes is due to increase of visiting points in the network. There might be some collision due to more than one source node competing for packet transfer. Still the results show better performance with $16 \%$ improvement compared with ICGS protocol.

\section{CONCLUSIONS}

In this paper, we have proposed an intelligent system which can find the better time frames for data collection in the next iterations. Tour length of MobiSink is calculated in prior with the help of history generated in the previous iteration. Source node locations are purely random and are shared to MobiSink which is first input to find the route points of the tour. Next is to find the duration of time to be spent at each point with stored information. ORPIS protocol is applied to find time frame duration at each route points. Once all time frames are obtained, MobiSink will form a vector and traverse at each node. Energy harvesting model is introduced which does renewal of energy to each node periodically. With energy harvesting, nodes will have flexibility sending packets with considering the expenditure of energy. This led to increase of reliability and thus the delivery ratio of the network. The protocol is compared with ICGS and IMPR protocols and results show that performance of proposed ORPIS protocol is better.

\section{REFERENCES}

1. M. Garey, and D. Johnson, "Computers and Intractability: AGuide tothe Theory of NP-Completeness", Bell Laboratories Incorporated, 1979.

2. T. H. Cormen, C. E. Leiserson, R. L. Rivest, and C. Stein, “Introduction to Algorithms", MIT Press, 3rd Edition, 2009.

3. W. Liang, J. Luo, and X. Xu. "Prolonging network lifetime via acontrolled mobile sink in wireless sensor networks". Proc. OfGLOBECOM, IEEE,2010.

4. M. D. Francesco, S. K. Das, and G. Anastasi, "Data collection inwireless sensor networks with mobile elements: a survey”, ACM Trans.on Sensor Netw., 2011. 
5. W. Liang and J. Luo. "Network lifetime maximization in sensornetworks with multiple mobile sinks." Proc.OfLCN'11, IEEE,2011.

6. S. Gao, H .Zhang, and S. K. Das, "Efficient data collection in wirelesssensor networks with path-constrained mobile sinks”,IEEE Trans. OnMobile Comput., vol.10,2011.

7. R. S. Liu, K. W. Fan, Z. Zheng and P. Sinha. "Perpetual and fair datacollection for environmental energy harvesting sensornetworks",IEEE/ACM Trans. on Netw., vol. 19, 2011.

8. X. Ren and W. Liang, "Delay-tolerant data gathering in energyharvesting sensor networks with a mobile sink,", in Proc. Of IEEEGLOBECOM Conference, 2012.

9. X. Ren, and W. Liang, "The use of a mobile sink for quality datacollection in energy harvesting sensor networks," in Proc. Of IEEEWireless Commun. and Netw. Conference (WCNC), 2013.

10. X. Ren, W. Liang, and W. Xu, "Use of a mobile sink for maximizingdata collection in energy harvesting sensor networks,"in Proc. of IEEE42nd Int. Conf. on Parallel Processing, 2013.

11. L. He, J. Pan and J. Xu, "A progressive approach to reducing datacollection latency in wireless sensor networks with mobile elements, "IEEE Trans. Mobile Comput., vol. 12, 2013.

12. Naai-Jung, Shih. "Experiencing an Informal Dining Route Through the 3D Scanned Data of Urban Fabric." Browser Download This Paper (2016).

13. D. Gunduz, K. Stamatiou, N. Michelusi, and M. Zorzi, ”DesigningIntelligent Energy Harvesting Communication Systems", IEEECommunications Magazine, vol. 52,2014.

14. J. Chen, S. He, and Y. Sun, "Rechargeable Sensor Networks:Technology, Theory, and Application", World ScientificPublishing,2014.

15. Mehrabi, and K. Kim, "Maximizing data collection throughput on apath in energy harvesting sensor networks $u$

\section{Authors}

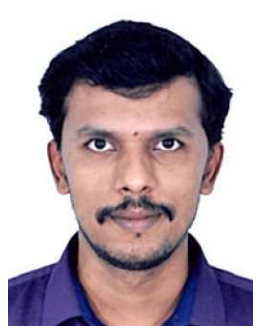

Kumar Swamy B.V received the B.E. and M.Tech. Degree from Visvesvaraya Technological University (V.T.U), Karnataka State, India in 2007 and 2009, respectively and a Ph.D research scholar at V.T.U. He is currently a research scholar at the Kalpataru Institute of Technology, Karnataka, India. His current research interests include intelligent networking, big data analytics and deep learning. He has filed for 5 patents in big data and application modernization areas. 


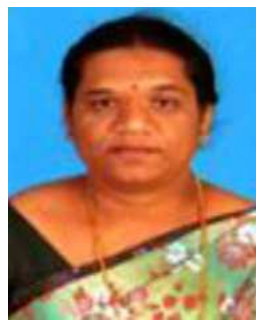

Dr. Gowramma Y.P received a B.E. degree from A.I.T, Chikmagalore, India in 1995 and M.Tech. Degree from N.I.T.K, Surathkkal, India in 2000 and Ph.D degree from Visvesvaraya Technological University, Karnataka State, India in 2014. She is currently working as Professor at the Kalpataru Institute of Technology, Karnataka, India. Her current research interests include image processing, computer networks and system analysis.

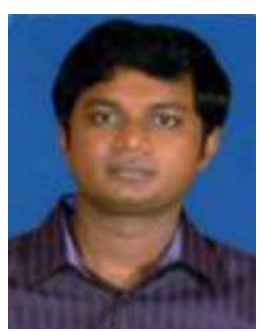

Ananda Babu J received the B.E. and M.Tech. Degree from Visvesvaraya Technological University, Karnataka State, India. in 2007 and 2009, respectively and a Ph.D research scholar at V.T.U. He is currently an Assistant Professor at the Kalpataru Institute of Technology, Karnataka, India. His current research interests include computer networks and wireless sensor networks. 
